\title{
Synthesis of condensed polynuclear aromatic resin from furfural extract oil of reduced-pressure route II
}

\author{
Wu Mingbo*, Jiang Wei, Wang Yuwei, Li Shibin and Zheng Jingtang** \\ State Key Laboratory of Heavy Oil Processing, China University of Petroleum, Qingdao, Shandong 266580, China \\ (C) China University of Petroleum (Beijing) and Springer-Verlag Berlin Heidelberg 2013
}

\begin{abstract}
As an industrial byproduct of oil refining, furfural extract oil from reduced-pressure route II with high aromatic content was used to prepare heat-resistant condensed polynuclear aromatic (COPNA) resin for the first time. The basic properties of furfural extract oil and the resultant COPNA resin were characterized by infrared spectroscopy (FT-IR), nuclear magnetic resonance spectroscopy $\left({ }^{1} \mathrm{H}-\mathrm{NMR}\right)$, thermogravimetric analysis (TGA) and elemental analysis (EA). The result showed that heat treated furfural extract oil was successfully used for the synthesis of heat-resistant COPNA resin. The average structural parameters of raw materials and prepared resin were calculated by the improved Brown-Ladner method, and the averaged molecular structure of the resin was obtained. The reaction mechanism for the synthesis of COPNA resin was suggested as an acid-catalyzed positive ion type polymerization.
\end{abstract}

Key words: Furfural extract oil, reduced-pressure route II, COPNA resin, synthesis, reaction mechanism

\section{Introduction}

The synthesis of COPNA resin was firstly reported by Otani (Otani et al, 1986). As a novel heat-resistant material with good lubricity, mechanical properties and mouldability, this resin has attracted considerable attention for its potential applications (Kusakabe et al, 1998; Li et al, 2010; Lin et al, 2010a; 2010b; Tanemura et al, 2011; 2012; Zhao et al, 2008). In the early stages, raw materials for the preparation of COPNA resin were confined to pure aromatic substances (Nakajima et al, 1995; Nawa, 1996; Nawa and Ohkita, 1997). Recently, a number of similar raw materials including coal derived and petroleum based mixtures have been successfully used to prepare COPNA resin (Guo et al, 2002; Li et al, 2008; Shi et al, 2012; Wu et al, 2012; 2012). Furfural extract oil from reduced-pressure route II, commonly used as fuel oil for boilers or for the manufacture of synthetic rubber, is a byproduct of oil refining. With a high aromatic content, narrow molecular weight distribution and low content of heavy metals, furfural extract oil is theoretically considered as an ideal candidate raw material to prepare COPNA resin.

In this work, furfural extract oil from reduced-pressure route II was firstly heated at atmospheric pressure to remove its volatile components. Then, COPNA resin was eventually synthesized through the reaction of the thermally condensed oil and the cross-linking agent (1, 4-benzenedimethanol, PXG), which was catalyzed by p-toluene sulfonic acid (PTS). The resultant resin as well as the raw materials

\footnotetext{
*Corresponding authors. email: wumb@upc.edu.cn;

**Corresponding author. email: jtzheng03@163.com

Received July 11, 2013
}

were characterized by elemental analysis (EA), infrared spectroscopy (FT-IR), nuclear magnetic resonance spectroscopy $\left({ }^{1} \mathrm{H}-\mathrm{NMR}\right)$ and thermogravimetric analysis (TGA).

\section{Experimental}

\subsection{Raw materials}

Furfural extract oil from reduced-pressure route II was provided by Jiangshan Polymer Material Company, China. PXG (AR) and PTS (AR) were both purchased from SINOPHARM Chemical Reagent Company, China. Toluene was obtained from Tianjin Chemical Reagent Company, China. Quinoline was purchased from Tingxin Chemical Reagent Company, China.

\subsection{Heat treatment of furfural extract oil from reduced-pressure route II}

Furfural extract oil from vacuum second side-cut was added into a reaction kettle equipped with a thermometer and mechanical stirrer. The reaction kettle was heated to $420{ }^{\circ} \mathrm{C}$ at $2{ }^{\circ} \mathrm{C} / \mathrm{min}$ in a nitrogen flow of $50 \mathrm{~mL} / \mathrm{min}$ and then kept for $3 \mathrm{~h}$. A small amount of the light fraction of furfural extract oil was found to have evaporated after heat treatment and the thermally condensed oil was obtained.

\subsection{Preparations of COPNA resin}

The thermally condensed oil, PXG and PTS (with a weight ratios of $75: 20: 5$ ) were fully mixed in a reactor, and then the mixture was stirred and heated in a nitrogen flow of $40 \mathrm{~mL} / \mathrm{min}$. The reactor was heated to $130{ }^{\circ} \mathrm{C}$ at $5{ }^{\circ} \mathrm{C} /$ 
min and kept for $1 \mathrm{~h}$, then heated to $150{ }^{\circ} \mathrm{C}$ and held until a solidified COPNA resin was observed. It is noteworthy that the viscosity of this system increased gradually, indicating that the degree of polymerization increased gradually during the reaction.

\subsection{Characterization}

The prepared COPNA resins and the raw materials were analyzed by TGA, FT-IR, NMR and elemental analysis.

For TGA measurements, about $10 \mathrm{mg}$ sample was placed in a platinum crucible of a SHIMADZU DTG-60 thermogravimetric analyzer, and heated from room temperature to $900{ }^{\circ} \mathrm{C}$ at a heating rate of $10{ }^{\circ} \mathrm{C} / \mathrm{min}$ under a nitrogen flow of $50 \mathrm{~mL} / \mathrm{min}$.

FT-IR spectra of the samples were obtained with a Shimadzu 8400S FT-IR spectrometer in transmittance mode. The scan frequency of each spectrum was $15 \mathrm{~s}^{-1}$ with a resolution of $0.85 \mathrm{~cm}^{-1}$.

A BRUKER AV $500{ }^{1} \mathrm{H}-\mathrm{NMR}$ spectrometer was used to characterize the state of different hydrogen in prepared COPNA resins and the raw materials. The solvent used was chloroform, the internal standard label was trimethylphenylsilane, the sweep length was $10,000 \mathrm{~Hz}$, and the resonance frequency was $500 \mathrm{MHz}$.

Elemental analysis was performed by the Vario EL organic element analyzer. The structural data of the obtained COPNA resin and raw materials was calculated by the Brown-Ladner formula (Wu et al, 2012a; 2012b).

\section{Results and discussion}

\subsection{Properties of furfural extract oil and thermally condensed oil}

The basic properties of furfural extract oil and its thermally condensed oil are given in Table 1 . We can see that the average molecular weight of furfural extract oil was 273 , the saturate content was $11.1 \mathrm{wt} \%$, while the aromatics content was $81.6 \mathrm{wt} \%$. After heat treatment, the average molecular weight was increased to 338 , the saturate content was reduced

Table 1 Properties of furfural extract oil and its thermally condensed oil

\begin{tabular}{ccc}
\hline Properties & Furfural extract oil & Thermally condensed oil \\
\hline M & 273 & 338 \\
CR, wt $\%$ & 8.9 & 18.2 \\
QI, wt $\%$ & 0.1 & 0.2 \\
TI, wt $\%$ & 0.3 & 0.6 \\
(TI-QI), wt $\%$ & 0.2 & 0.4 \\
Saturates, wt $\%$ & 11.1 & 3.7 \\
Aromatics, wt $\%$ & & 4.1 \\
Light & 21.5 & 34.3 \\
Middle & 37.9 & 43.8 \\
Heavy & 22.2 & 14.1 \\
Others, wt $\%$ & 7.3 &
\end{tabular}

Notes: $\mathrm{M}$, average molecular weight; $\mathrm{CR}$, carbon residue ratio; TI, toluene insoluble; QI, quinoline insoluble. to $3.7 \mathrm{wt} \%$ and the light aromatics content was decreased to $4.1 \mathrm{wt} \%$. However, there was a remarkable increase in heavy aromatics, from $22.2 \mathrm{wt} \%$ of furfural extract oil to $43.8 \mathrm{wt} \%$ of thermally condensed oil. TI and QI values of thermally condensed oil were both higher than those of furfural extract oil. These results showed that polymerization reactions took place during the process of heat treatment.

Table 2 shows the elemental analysis of furfural extract oil and thermally condensed oil. After the thermal condensation of furfural extract oil, the total content of other elements was reduced from $2.6 \mathrm{wt} \%$ to $1.7 \mathrm{wt} \%$, the value of $\mathrm{C} / \mathrm{H}$ increased from 1.10 to 1.16 , and the $f_{\mathrm{A}}$ data increased from 0.68 to 0.76 . The results indicated that some ingredients in furfural extract oil can be removed by heat treatment and condensation in this process.

Table 2 Elemental analysis of furfural extract oil and its thermally condensed oil

\begin{tabular}{cccccccc}
\hline & \multicolumn{7}{c}{ Elements, wt \% } \\
\cline { 2 - 7 } Samples & $\mathrm{C}$ & $\mathrm{H}$ & $\mathrm{N}$ & $\mathrm{O}$ & Others & $f_{\mathrm{A}}$ \\
\hline $\begin{array}{c}\text { Furfural extract } \\
\text { oil }\end{array}$ & 89.5 & 6.8 & 0.3 & 0.8 & 2.6 & 1.10 & 0.68 \\
$\begin{array}{c}\text { Thermally } \\
\text { condensed oil }\end{array}$ & 90.4 & 6.5 & 0.6 & 0.8 & 1.7 & 1.16 & 0.76 \\
\hline
\end{tabular}

\subsection{Properties of COPNA resin}

\subsubsection{Basic properties of COPNA resin}

Table 3 shows the basic properties of the obtained COPNA resin. Tables 1 and 3 indicate that the residual carbon ratio was dramatically increased from $18.2 \mathrm{wt} \%$ in thermally condensed oil to $32.6 \mathrm{wt} \%$ in the COPNA resin. Both TI and QI values were also greatly increased, and the TI-QI value was increased significantly from $0.4 \mathrm{wt} \%$ in the thermally condensed oil to $12.4 \mathrm{wt} \%$ of COPNA resin. The TI-QI value is a critical parameter that determines the binding performance, and resin with a TI-QI value of $>8.0 \mathrm{wt} \%$ is

Table 3 Properties of COPNA resin

\begin{tabular}{cc}
\hline Properties & Values \\
\hline M & 1412 \\
QI, wt $\%$ & 32.6 \\
TI, wt $\%$ & 5.6 \\
(TI-QI), wt $\%$ & 17.9 \\
Softening point, ${ }^{\circ} \mathrm{C}$ & 12.4 \\
C, wt $\%$ & 110 \\
H, wt $\%$ & 89.1 \\
N, wt $\%$ & 6.1 \\
O, wt $\%$ & 0.4 \\
Others, wt $\%$ & 1.5 \\
C $/ \mathrm{H}$ & 2.9 \\
$f_{\mathrm{A}}$ & 1.22 \\
\hline
\end{tabular}


deemed suitable for industrial application as a binder. In addition, the average molecular weights in Tables 1 and 3 showed that the average degree of polymerization was around 3.

\subsubsection{FT-IR analysis}

The FT-IR spectra of furfural extract oil, thermally condensed oil and COPNA resin are shown in Fig. 1. Peaks at $3,420-3,550 \mathrm{~cm}^{-1}$ in Fig. 1 were attributed to N-H stretching vibration and $\mathrm{O}-\mathrm{H}$ stretching vibration. Peaks at $3,046 \mathrm{~cm}^{-1}$ and $1,602 \mathrm{~cm}^{-1}$ were attributed to aromatic $\mathrm{C}-\mathrm{H}$ and $\mathrm{C}=\mathrm{C}$ stretching vibrations. The peak at $1,455 \mathrm{~cm}^{-1}$ was from $\mathrm{C}-\mathrm{H}$ bending vibration. These peaks clearly revealed the existence

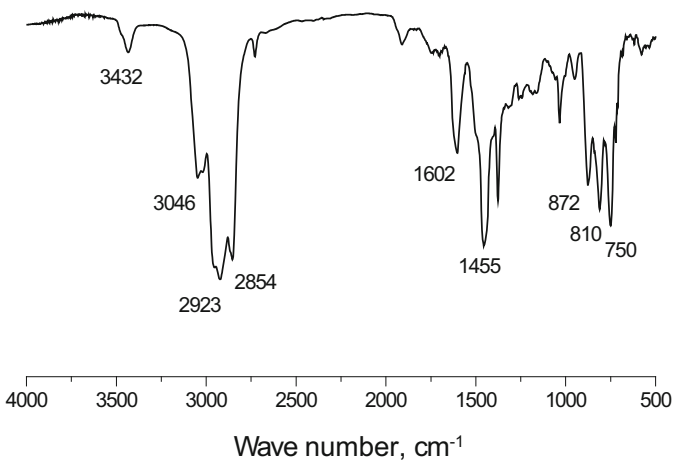

(a)
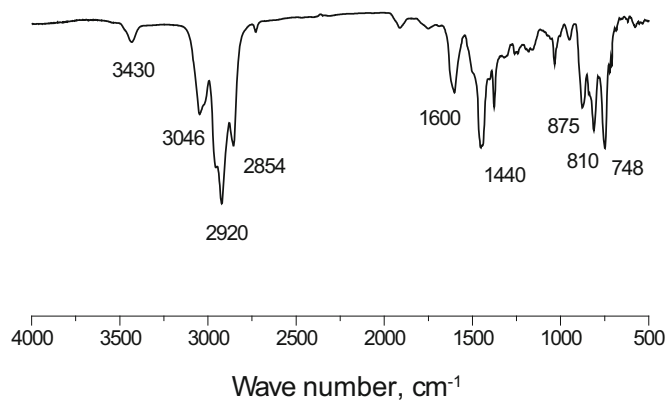

(b)
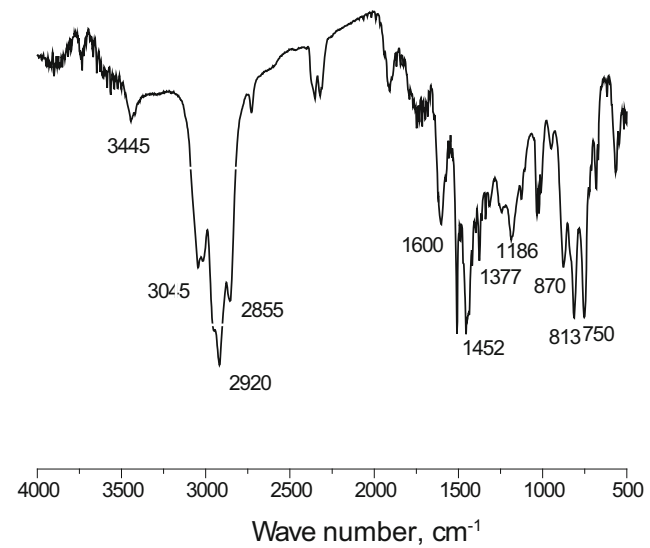

(c)

Fig. 1 FT-IR spectra of furfural extract oil (a), thermally condensed oil (b) and COPNA resin (c) of the elements $\mathrm{C}, \mathrm{H}, \mathrm{N}$ and $\mathrm{O}$, as well as the corresponding chemical bonds they formed. The peaks at $750 \mathrm{~cm}^{-1}, 810$ $\mathrm{cm}^{-1}$ and $872 \mathrm{~cm}^{-1}$ were possibly generated by the out-ofplane bending vibrations of 2, 3, 4-aromatic $\mathrm{C}-\mathrm{H}$. These results confirmed the existence of various forms of aromatic substitution reaction.

In contrast with peaks in Fig.1(a), peaks at 2,920 and $2,854 \mathrm{~cm}^{-1}$ attributed to the $\mathrm{C}-\mathrm{H}$ stretching vibration in $-\mathrm{CH}_{2}-$ were weakened in Fig. 1(b). The peaks between 900 $\mathrm{cm}^{-1}$ and $650 \mathrm{~cm}^{-1}$ in Fig. 1(b) (such as $875 \mathrm{~cm}^{-1}, 810 \mathrm{~cm}^{-1}$ and $748 \mathrm{~cm}^{-1}$ ) attributed to out-of-plane bending vibration of $\mathrm{C}-\mathrm{H}$ in aromatics were weaker than those of Fig. 1(a). These changes indicated that condensation of furfural extract oil occurred during heat treatment.

In Fig. 1(c), the absorption peaks around 2,920 and 2,855 $\mathrm{cm}^{-1}$ were stronger than those of Fig. 1(b), indicating the occurrence of the cross-linking reactions between PXG and thermally condensed oil. The peaks in Fig. 1(c) at 1,600 $\mathrm{cm}^{-1}$ attributed to $\mathrm{C}=\mathrm{C}$ stretching vibration and the peaks between $900 \mathrm{~cm}^{-1}$ and $650 \mathrm{~cm}^{-1}$ belonged to out-of-plane bending vibration of $\mathrm{C}-\mathrm{H}$ in aromatics were much stronger than those of Fig. 1(b), indicating that the cross-linking reactions changed the types of aromatic substitution.

\subsection{3 ${ }^{1} \mathrm{H}-\mathrm{NMR}$ analysis}

The ${ }^{1} \mathrm{H}-\mathrm{NMR}$ spectra of furfural extract oil, thermally condensed oil and COPNA resin are shown in Fig. 2. The large peaks between $\delta 7.0$ and $\delta 9.0$ were attributed to the absorption resonance of hydrogen in aromatic rings. Fig. 2 also presents $\alpha-\mathrm{H}$ absorption resonance peaks between $\delta 2.0$ and $\delta 4.0, \beta-\mathrm{H}$ absorption resonance peaks between $\delta 1.0$ and $\delta 2.0$, as well as $\gamma-\mathrm{H}$ absorption resonance peaks between $\delta 0.5$ and $\delta$ 1.0. The data of Fig. 2 is summarized in Table 4, where $\mathrm{H}_{\alpha}$ means the weight ratio of $\alpha-\mathrm{CH}_{3}, \alpha-\mathrm{CH}_{2}$ and $\alpha-\mathrm{CH}$ in aromatic ring (i.e. the weight ratio of $\alpha$-hydrogen to the total hydrogen in weight). $\mathrm{H}_{\mathrm{A}}$ refers to the weight ratio of aromatic $\mathrm{H}$ in aromatic rings. Similarly, $\mathrm{H}_{\beta}$ and $\mathrm{H}_{\gamma}$ refer to the weight ratio of $\beta-\mathrm{CH}_{2}$ and $\beta-\mathrm{CH}$ hydrogen and $\gamma-\mathrm{CH}_{3}$ hydrogen in the aromatic rings, respectively.

It is noteworthy that the content of $\mathrm{H}_{\mathrm{A}}$ increased from $29.6 \%$ of furfural extract oil to $43.9 \%$ of thermally condensed oil, while there was a distinct decrease in the content of $\mathrm{H}_{\beta}$. This was because $\beta$-H was mostly located on side-chains, and the decrease in $\beta$-H demonstrated side-chain cleavage and an increase in polymerization degree, which was in accordance with the analysis of aromatics and the results of FTIR. The content of $\mathrm{H}_{\alpha}$ increased significantly after synthetic reaction, indicating that the aromatics connected with $-\mathrm{CH}_{2}-$ of PXG.

\subsection{Mechanism of COPNA resin synthesis}

Based on the results of FTIR, ${ }^{1} \mathrm{H}-\mathrm{NMR}$, elemental analysis and average molecular weight analysis of furfural extract oil, thermally condensed oil and COPNA resin, the average structural parameters, including $\mathrm{C}_{\mathrm{T}}, \mathrm{H}_{\mathrm{T}}, \mathrm{f}_{\mathrm{A}}, \mathrm{H}_{\mathrm{AU}} / \mathrm{C}_{\mathrm{A}}, \sigma, \mathrm{R}_{\mathrm{T}}, \mathrm{R}_{\mathrm{A}}$, $R_{N}$, and $C_{R}$, were obtained by the improved Brown-Ladner method. The obtained average structure parameters are shown in Table 5, in which the $\mathrm{S}, \mathrm{O}$ and $\mathrm{N}$ levels were negligible. The reaction mechanism and averaged molecular structure of COPNA resin are as follows: 


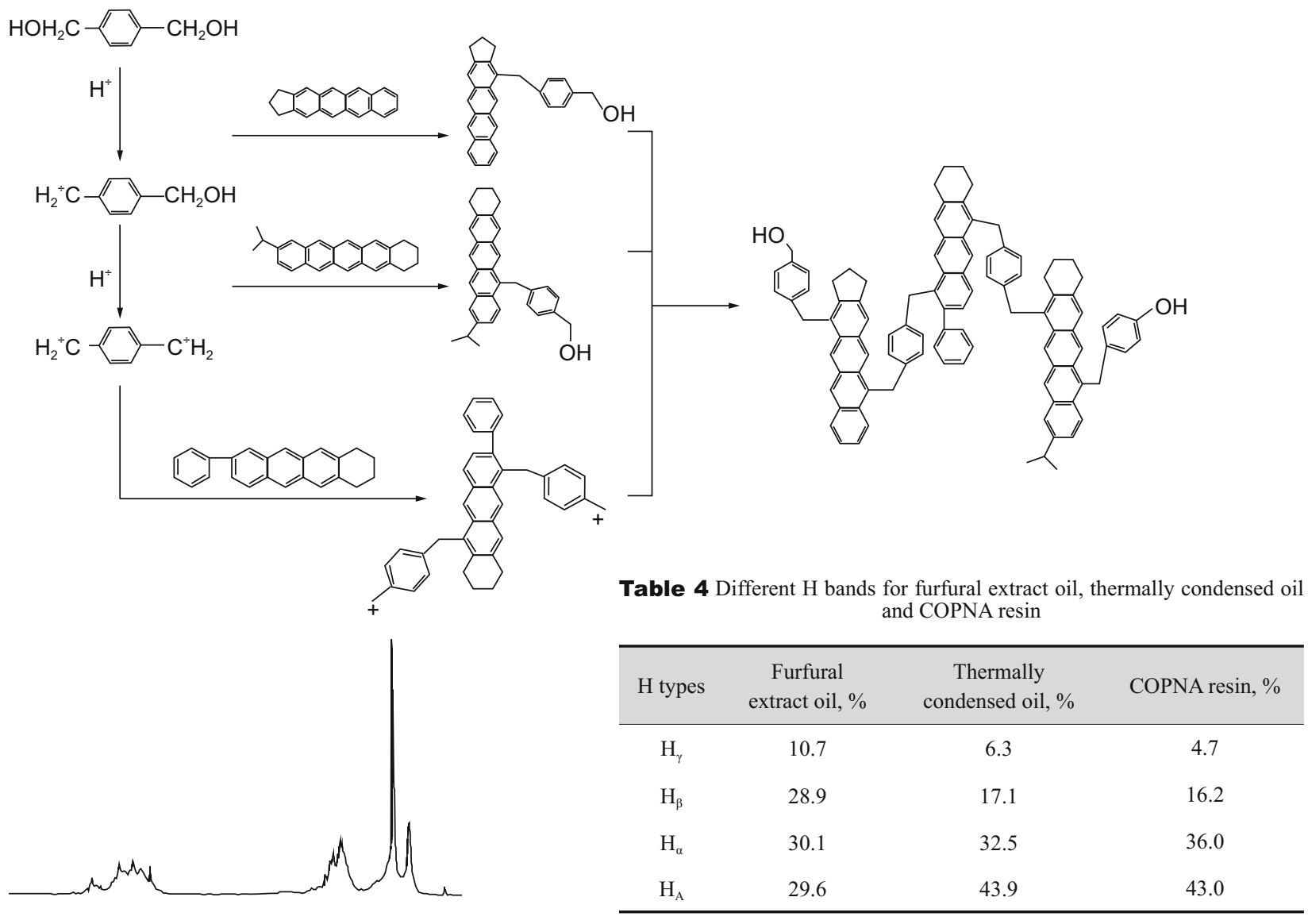

Table 5 Average molecular structural parameters of furfural extract oil, thermally condensed oil and COPNA resin

\begin{tabular}{cccccccc}
\hline Samples & $\mathrm{C}_{\mathrm{T}}$ & $\mathrm{H}_{\mathrm{T}}$ & $\mathrm{H}_{\mathrm{AU}} / \mathrm{C}_{\mathrm{A}}$ & $\Sigma$ & $\mathrm{R}_{\mathrm{T}}$ & $\mathrm{R}_{\mathrm{A}}$ & $\mathrm{R}_{\mathrm{N}}$ \\
\hline $\begin{array}{c}\text { Furfural extract oil } \\
\begin{array}{c}\text { Thermally } \\
\text { condensed oil }\end{array}\end{array}$ & 20.36 & 18.56 & 0.60 & 0.34 & 5.14 & 3.00 & 2.14 \\
$\begin{array}{c}\text { COPNA resin } \\
\text { COPN }\end{array}$ & 21.97 & 0.68 & 0.27 & 5.80 & 4.34 & 1.46 \\
\hline
\end{tabular}

\subsection{Heat resistance analysis}

The thermal decomposition behavior of furfural extract oil, thermally condensed oil, and COPNA resin are shown in Fig. 3. The furfural extract oil had a significant weight loss even at $50{ }^{\circ} \mathrm{C}$, while the degradation onset temperature of thermally condensed oil was above $200{ }^{\circ} \mathrm{C}$, demonstrating that most light fractions were removed during thermal condensation. Compared with the weight loss of thermally condensed oil, the weight loss of COPNA resin was almost unchanged in the initial stage, and its loss rate was relatively slow after $250{ }^{\circ} \mathrm{C}$ in comparison with those of furfural extract oil and thermally condensed oil. When the temperature was raised to $500{ }^{\circ} \mathrm{C}$, the weight left of COPNA resin still remained $31.5 \mathrm{wt} \%$, much higher than $12.0 \mathrm{wt} \%$ of thermally condensed oil.

\section{Conclusions}

With short side chains, lower steric hindrance, high aromatic content and purity, furfural extract oil from reducedpressure route II is selected as raw material for the preparation 


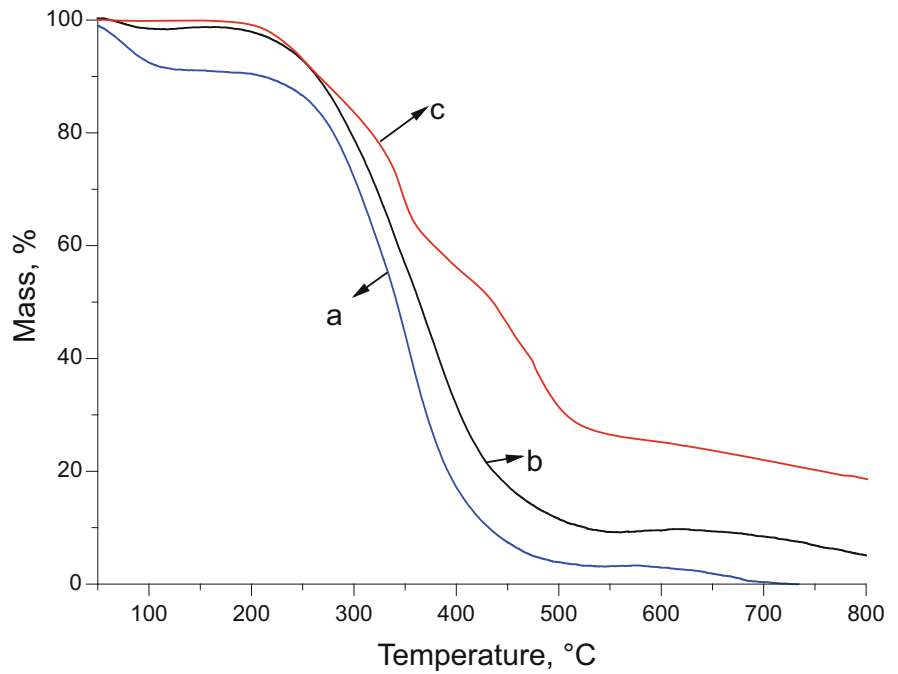

Fig. 3 TGA curves of furfural extract oil (a), thermally condensed oil (b) and COPNA resin (c)

of COPNA resin. After heat treatment, the polymerization degree has increased and side-chain cleavage occurred and the content of heavy aromatics has increased remarkably. COPNA resin has been successfully prepared from thermally condensed oil with PXG as a cross-linking agent and PTS as catalyst. In order to get a clearer understanding of the condensation and polymerization mechanisms, the average molecular structures of raw material and COPNA resin are obtained based on the results of FTIR, ${ }^{1} \mathrm{H}-\mathrm{NMR}$, element analysis and average molecular weight analysis. The degree of polymerization is found to be around 3 . The resulting COPNA resin with high heat resistance is a potential binder in carbon material preparation.

\section{Nomenclatures}

$\mathrm{C}_{\mathrm{T}} \quad$ Total average carbon number

$\mathrm{H}_{\mathrm{T}} \quad$ Total average hydrogen number

$\mathrm{f}_{\mathrm{A}} \quad$ Ratio of aromatic carbon number to total carbon number

$\mathrm{H}_{\mathrm{AU}} / \mathrm{C}_{\mathrm{A}} \quad$ Average condensation degree

$\sigma \quad$ Hydrogen replaced rate surrounding aromatic rings of average molecular

$\mathrm{R}_{\mathrm{T}} \quad$ Total average rings

$\mathrm{R}_{\mathrm{A}} \quad$ Average aromatic rings

$\mathrm{R}_{\mathrm{N}} \quad$ Average naphthenic rings

$\mathrm{C}_{\mathrm{R}} \quad$ Average carbon number on the rings

\section{Acknowledgements}

This work is financially supported by the National Natural Science Foundation of China (Nos. 20876176, 51172285, 51372277), the Natural Science Foundation of Shandong Province (ZR2011EL030), and the Fundamental Research
Funds for the Central Universities (14CX02060A).

\section{References}

Guo Y S, Zha Q F and Wu M B. Synthesis of condensed polynuclear aromatic resins from aromatics-enriched feedstocks. Carbon Techniques. 2002. 119(02): 10-14 (in Chinese)

Kusakabe K, Gohgi S and Morooka S. Carbon molecular sieving membranes derived from condensed polynuclear aromatic (COPNA) resins for gas separations. Industrial and Engineering Chemistry Research. 1998. 37(11): 4262-4266

Li J T, Lin Q L, Ke Y L, et al. Rheological behavior of the copolymer resin of ally-COPNA resin and BMI. Polymer Materials Science and Engineering. 2010. 26(5): 54-56 (in Chinese)

Li S B, Song C G and Hou B H. Study of the synthesis and composite properties of condensed poly-nuclear aromatics resin using coal tar pitch as monomer and trioxane as cross-linking agent. Carbon. 2008. 136(4): 13-17 (in Chinese)

Lin Q L, Zheng R G and Tian P H. Preparation and characterization of BMI resin/graphite oxide nanocomposites. Polymer Testing. 2010a. 29(5): 537-543

Lin Q L, Zheng M Z, Qin T, et al. Preparation of solid carbon spheres by pyrolysis of allyl COPNA-BMI resin. Journal of Analytical and Applied Pyrolysis. 2010b. 89(1): 112-116

Nakajima S, Iijima Y, Inoue T, et al. Anomalous magnetic properties of poly-condensate of fused polynuclear aromatics (COPNA) resin. Synthetic Metals. 1995. 71(1): 1817-1818

Nawa K. Development of flexible polymer blend films from advanced COPNA resin and Nylon 6. Journal of Applied Polymer Science. 1996. 61(10): 1737-1746

Nawa K and Ohkita M. Reliability of the laminate from advanced COPNA-resin/E-glass fabrics system. IEEE Transactions on Components, Packaging, and Manufacturing Technology, Part B. 1997. 20(1): 78-86

Otani S, Ota L and Raskovie V. Carbonization behavior of condensed poly-nuclear aromatic (COPNA) resins. Carbon. 1986. 127: 162-170 (In Japanese)

Shi Y Y, Wu M B, Li S B, et al. Effects of cross-linking agents on synthesis of heat-resistant resin from ethylene tar. Applied Mechanics and Materials. 2012. 217-219: 1159-1165

Tanemura K, Suzuki T, Nishida Y, et al. Synthesis of the sulfonated condensed polynuclear aromatic (S-COPNA) resins as strong protonic acids. Tetrahedron. 2011. 67(6): 1314-1319

Tanemura K, Suzuki T, Nishida Y, et al. Synthesis of the strongly acidic sulfonated condensed polynuclear aromatic (S-COPNA) resins using aromatic aldehydes as cross-linking agents. Polymer Bulletin. 2012. 68(3): 705-719

Wu M B, Shi Y Y, Li S B, et al. Synthesis and characterization of condensed poly-nuclear aromatic resin using tiroxane as crossinglinking agent. New Carbon Materials. 2012a. 27(6): 469-475

Wu M B, Shi Y Y, Li S B, et al. Synthesis and characterization of condensed poly-nuclear aromatic resin derived from ethylene tar. China Petroleum Processing and Petrochemical Technology. 2012b. 14(4): 21-26

Zhao J B, Lin Q L, Chen Y, et al. Synthesis and properties of allylCOPNA resin. Polymer Materials Science and Engineering. 2008. 24(11): 51-53 (in Chinese)

(Edited by Zhu Xiuqin) 